\title{
Un modèle pour comprendre le cycle de vie des communautés touristiques transnationales
}

\author{
Rémy Tremblay \\ INRS-Urbanisation, culture et société
}

\section{Introduction}

Les rapports entre le tourisme et l'ethnicité font l'objet d'un nombre croissant d'études. Depuis une trentaine d'années, les chercheurs spécialistes de cet aspect particulier des études touristiques se sont surtout concentrés sur le tourisme ethnique, bien qu'un nombre croissant de chercheurs se penchent sur les activités touristiques découlant de groupes ethniques s'étant exilés de leur pays d'origine pour des raisons politiques majeures.

Le tourisme ethnique se décrit le plus souvent comme un type de tourisme dans lequel le visiteur, généralement originaire d'un pays industrialisé et à la recherche d'expériences exotiques, se rend dans un pays afin d'y passer un séjour dans un milieu habité par un groupe ethnoculturel soi-disant « ethnique ${ }^{1}$. Certains, parmi ceux qui pratiquent cette forme de tourisme, sont parfois qualifiés de « voyeuristes » vu leur comportement « immoral» à l'égard de leurs hôtes. On pense, entre autres, aux touristes qui visitent les aborigènes du "Outback» australien ou les Inuits de l'Arctique canadien.

Cependant, il est possible de prêter une définition différente au tourisme ethnique. En effet, les communautés ethniques situées dans les milieux urbains des pays occidentaux constituent, elles aussi, des lieux caractéristiques $\mathrm{du}$ tourisme ethnique. Pensons, par exemple, aux quartiers chinois de San Francisco ou italien de New York. Encore une fois, ces communautés hautement médiatisées sont souvent victimes de voyeuristes.
Mais un nombre croissant de communautés ethniques plus récentes servent de lieux de rencontre et d'échanges entre les nouveaux arrivants et les visiteurs du pays d'origine. Les communautés haïtiennes de Montréal et de Miami et les dizaines de communautés mexicaines des États-Unis illustrent parfaitement ce que Peggy Levitt ${ }^{2}$ appelle des « communautés transnationales ». Comme l'indiquent Levitt et Schiller ${ }^{3}$ : " Once we rethink the boundaries of social life, it becomes clear that the incorporation of individuals into nation-states and transnational connections are not contradictory social processes. Simultaniety is a possibility that needs to be theorized and explored. Rather than viewing migration as a one-way process, increasing numbers of scholars now recognize that migrants simultaneously live aspects of their lives in their sending countries at the same time that they are incorporated into the countries that receive them. Migrant incorporation into a new land and transnational connections to a homeland or to dispersed networks of family, compatriots, or persons who share a religious or ethnic identity can occur at the same time and reinforced each other ».

Si les mécanismes socioculturels des communautés transnationales semblent avoir été cernées, ceux relatifs à son rôle touristique demeurent moins connus. Le but de cet article vise justement à lever le voile sur les dimensions touristiques de la communauté transnationale. En s'inspirant d'une étude de cas des Québécois dans le sud-est de la Floride, nous proposerons un modèle permettant d'illustrer la dynamique qui est la base de la transformation d'un destination touristique de masse en une communauté touristique transnationale. 


\section{Les Québécois sur la côte sud-est de la Floride ${ }^{4}$}

Tandis que les ouvrages sur l'Amérique française abondent, les travaux portant sur la présence québécoise en Floride sont quasi inexistants. Rémy Tremblay est un des rares à avoir écrit sur ce sujet ${ }^{5}$.

D'emblé, ce que nous appellerons ici « Floribec» se réfère à l'espace des migrants et touristes du Québec, dont la majorité appartiennent à la classe ouvrière et dont les activités économiques et la vie quotidienne sont principalement orientées vers le tourisme de langue française et de culture québécoise. L'espace assez flou de Floribec s'étend sur près de cinq kilomètres dans les villes d'Hollywood, de Dania et de Hallandale, lesquelles se situent en banlieue Est de la région métropolitaine de Miami. D'après nos recherches sur le terrain ${ }^{6}$, c'est dans ces trois villes que se situent la majorité des commerces offrant des produits et services destinés aux immigrants et aux touristes québécois. Selon le recensement américain, la métropole de Miami comptait en l'an 2000 environ 25000 résidents d'origine québécoise (sans compter les nombreux illégaux). Il n'existe pas de source statistique précise, mais la côte sud-est floridienne attirerait annuellement entre 200000 et 400000 touristes québécois, en faisant de loin la première destination touristique des Québécois.

\section{D'après le recensement américain, la métropole de Miami comptait en I'an 2000 environ 25000 résidents d'origine québécoise.}

L'organisation spatiale de Floribec repose d'abord sur ses lieux structurants (motels, restaurants, bars, clubs, etc.). On a remarqué que la communauté ethnotouristique de Floribec, qui a véritablement pris naissance dans les années 1970, se forme autour d'un district des affaires récréationnelles (situé le long de la plage de Hollywood) regroupant ses plus importants lieux structurants et d'un centre ayant une importante population floribécoise et des services moins orientés vers le tourisme. Au-delà de ce centre, on trouve une périphérie où la population floribécoise, de même que les quelques points d'ancrage de la vie quotidienne, se diluent dans la culture anglosaxonne. La périphérie se caractérise également par le fait qu'elle est animée par des Floribécois ne ressentant pas d'attachement véritable pour la culture floribécoise, même s'ils entretiennent des rapports économiques étroits avec le centre de la communauté.

Par ailleurs, il est important d'ajouter que la communauté de Floribec, bâtie surtout autour de la langue et de la culture, entretient des liens étroits avec le Québec mais assez peu avec les "locaux». Le tourisme québécois joue un rôle déterminant dans le maintien de la vie communautaire floribécoise, mais les télécommunications ne peuvent dorénavant être ignorées. En fait, les NTIC font office d'institution sociale à distance. Certes, la télévision est avant tout un service destiné aux touristes québécois, et les commerçants floribécois sont conscients que ce type de service est vital pour attirer la clientèle visée. Mais tous les Floribécois, de même que les touristes québécois, en tirent profit puisqu'elle est vitale pour eux. Bref, selon nos recherches à Floribec, il est clair que les télécommunications créent un lien instantané et significatif au plan culturel entre le foyer d'origine et celui nouvellement adopté. Il s'agit d'institutions non physiques, le réseau social de base des communautés transnationales. Enfin, la communauté transnationale floribécoise est en voie de disparition. En effet, depuis environ cinq ans, les autorités municipales d'Hollywood et de Hallandale s'acharnent, avec succès, à faire disparâ̂tre Floribec, préférant attirer une clientèle plus raffinée et plus aisée. De plus, un nombre croissant de destinations tropicales bon marché s'offre aux Québécois, et ceux-ci les adoptent de plus en plus. C'est le cas, entre autres, de la République Dominicaine et de Cuba. Il s'agit-là d'une phase typique du cycle de vie de nombreuses stations balnéaires.

Ce bref tour d'horizon des communautés touristiques britanniques en Espagne et québécoise en Floride montre clairement qu'il s'agit, comme l'indique Levitt, de communautés de type transnationale : dans les deux cas, le maintien de la vie quotidienne dans la langue maternelle et les échanges avec la mère patrie sont fondamentaux. Mais comment une «destination soleil » devient-elle une communauté touristique transnationale?

\section{Modéliser le tourisme transnational}

Le géographe américain Dallen Timothy ${ }^{7}$ a proposé un modèle intitulé « Urban Ethnic Islands Created by 
Tourism » (tableau 1). Ce modèle, inspiré des études de Timothy dans la Petite Finlande à Lake WorthLantana, en Floride, montre que les îlots ethniques en milieu urbain issus du tourisme (Tourism-Created Urban Ethnic Islands) sont soit le produit de l'immigration, tel qu'évoqué par Peggy Levitt et par sa propre étude, soit le résultat du tourisme de masse, comme c'est le cas des études de cas décrites plus haut. Le tableau 1 illustre la réflexion de Timothy. Dans ce modèle, deux scénarios sont possibles, mais ils conduisent vers un même résultat (deuxième étape): l'émergence de ce que l'auteur appelle un îlot ethnique en milieu urbain créé par le tourisme, communauté ethnique touristique disposant de loisirs et de services suffisants pour que leur quotidien se déroule dans leur langue et dont lesquels sont perceptibles dans le paysage bâti. La première étape des scénarios A et B diverge selon qu'il s'agisse d'une enclave ethnique ou touristique, mais tous deux se développeront en un îlot ethnique en milieu urbain créé par le tourisme. Dans le cas du scénario A, la première étape se réfère au processus connu de mise en place d'une communauté ethnique, tandis que dans le scénario B, cette étape initiale illustre l'évolution d'une destination touristique visitée par un groupe ethno-linguistique étranger à la société d'accueil à partir de l'arrivée massive des touristes jusqu'au «marquage géosymbolique » de l'espace adopté par ces derniers.

\section{Tableau 1 - Îlots ethniques en milieu urbain issus du tourisme : deux scénarios Scénario A: Îlot ethnique touristique né de l'immigration}

Première étape : enclave ethnique urbaine

\section{- Arrivée des immigrants}

- Formation du regroupement ethnique

- Développement du paysage culturel

- Spécialisation des loisirs et des services

- Préservation du langage et des traditions
Deuxième étape : îlot ethnique en milieu urbain créé par le tourisme
- Les immigrants sont les touristes

- Le regroupement ethnique est le district touristique

- Le paysage culturel est le paysage touristique

- Les services sont spécialisés pour les résidents locaux et les touristes

- La langue et les activités culturelles au sein de l'enclave sont aussi ceux des touristes

\section{Scénario B: îlot ethnique touristique né du tourisme}

\section{Première étape : destination touristique}

- Arrivée des touristes

- Formation du district touristique

- Développement du paysage touristique

- Services spécialisés pour les touristes

- Apprentissage de la langue des touristes par les résidents
Deuxième étape: îlot ethnique en milieu urbain issu du tourisme
- Les immigrants sont les touristes

- Le regroupement ethnique est le district touristique

- Le paysage culturel est le paysage touristique

- Les services sont spécialisés pour les résidents locaux et les touristes

- La langue et les activités culturelles au sein de l'enclave sont aussi ceux des touristes
Même s'il ne s'agit que d'un modèle relativement statique et qu'il ne peut représenter avec exactitude toutes les nuances de l'évolution ethno-touristique de Floribec, nous considérons que le scénario B s'en rap- proche de très près. Cependant, les deux scénarios ne tiennent pas compte d'un aspect fondamental auquel le géographe Richard Butler ${ }^{8}$ s'était attardé : le cycle de vie et le déclin possible des destinations touristi- 
ques. Comme nous l'avons mentionné, la communauté floribécoise est sur le point de disparaître. Nous aimerions donc proposer une troisième étape au scénario B du modèle de Timothy : celle du déclin.

\section{Tableau 2 : Mise en tourisme des communautés transnationales vue à travers Floribec}

\section{Première étape : Adoption d'une destination touristique}

- Arrivée des touristes

- Formation du district touristique

- Développement du paysage touristique

- Services spécialisés pour les touristes

- Les résidents apprennent la langue des touristes

\section{Deuxième étape: Transformation de la destination en communauté ethnique}

- Les immigrants sont les touristes

- Le regroupement ethnique est le district touristique

- Le paysage culturel est le paysage touristique

- Les services sont spécialisés pour les résidents locaux et les touristes

- La langue et les activités culturelles au sein de l'enclave sont aussi ceux des touristes

- Les NTIC ont un rôle déterminant

\section{Troisième étape : Déclin de la communauté ethnique touristique transnationale}

- Déplacement dans l'espace du district touristique

- Perte de popularité de la communauté auprès des touristes

- Fermeture et/ou démolition de services

- Disparition de la communauté

Puisqu'un certain pattern dans la mise en tourisme des communautés issues du tourisme semble exister, nous aimerions proposer notre propre modèle explo- ratoire que nous conviendrons d'appeler " mise en tourisme des communautés transnationales » (tableau $3)$.

\section{Tableau 3 : Mise en tourisme de la communauté transnationale}

\section{Adoption}

Un groupe ethno-linguistique transfrontalier adopte de façon massive une destination touristique.

\section{Transformation}

Les apports touristique et immigrationnel constants, les NTIC et la mise sur pied de services transforment une destination touristique en communauté ethnique touristique transnationale.

\section{Déclin}

La compétition d'autres destinations touristiques, divers phénomènes sociaux, culturels ou urbains causent le déclin d'une communauté ethnique touristique transnationale. 
Ce modèle compte trois étapes. La première fait référence à l'adoption d'une destination touristique assez délimitée dans l'espace (ville, région, etc.) par un groupe ethno-linguistique distinct de celui du pays ou de la région hôte, Deuxièmement, une transformation s'établit. Il ne s'agit plus simplement d'une destination de masse, mais bien d'une communauté ethnique dont l'économie et la vie quotidienne dépendent de rapports étroits et constants avec le pays d'origine (touristes, migrants et NTIC). En troisième lieu, il y a une phase de déclin. Les motifs peuvent être très variés tout comme les effets. Certaines communautés touristiques transnationales peuvent ne pas subir de déclin. Néanmoins, l'expansion ne peut être continue et il y aura nécessairement stagnation, décroissance économique, modification du style de vie (loisirs et services) et/ou déplacement dans l'espace de la communauté.

\section{Conclusion}

Il va sans dire que tout modèle ne colle pas totalement à la réalité et qu'il demeure perfectible. Cependant, nous croyons que le modèle suggéré, inspiré de celui de Timothy, constituera un outil non négligeable pour comprendre, dans une perspective touristique, les comportements socio-spatiaux et migratoires d'autres groupes similaires au nôtre et aussi de ceux des réfugiés politiques et des groupes marginalisés et victimes de ségrégation. Le modèle pourrait également éclairer sur les réseaux sociaux et services relativement à la migration touristique des occidentaux dans les pays en voie de développement et du tiers-monde.

\section{Notes et références}

1 Hitchcock, M. (2001). «Anthropological Reflects on the Study of Tourism », dans G, Wall (dir.), Contemporary Perspectives on Tourism, Waterloo, Department of Geography, Occasional Paper Number 17, University of Waterloo, p. 109-128; King, B. (1994). " What is Ethnic Tourism? An Australian Perspective », Tourism Management, vol. 15, no 3, p. 173-176; Ostrowski, S. (1991). «Ethnic Tourism : Focus on Poland», Tourism Management, vol. $12, \mathrm{n}^{\mathrm{o}} 2$, p. $125-131$.

2 Levitt, P. (2001). The Transnational Villagers, Berkeley, University of California Press.

3 Levitt, P. et N.G. Schiller (2003). « Transnational Perspectives on Migration : Conceptualizing Simultaneity », Draftwork in Progress, p. 3.

4 Le but ici n'est pas de tracer un profil détaillé de la communauté transnationale touristique québécoise en Floride.

5 Entre autres: Tremblay, R. (2000). «Explorer la Floride canadienne-française », dans A. Fauchon (dir.), La francophonie panaméricaine : état des lieux, Winnipeg, Presses universitaires de Saint-Boniface, p 129-151; Tremblay, R. (2001). Floribec ou les Québécois en vacances, Montréal, INRS-urbanisation, culture et société; Tremblay, R. (2003). « Le déclin de Floribec », Téoros, vol. 22, $\mathrm{n}^{\mathrm{o}} 1, \mathrm{p} .18-27$.

6 Tremblay (2001), op. cit.

7 Timothy, D. (2002) « Tourism and the Growth of Tourism Ethnic Islands », dans M. Hall and A. Williams (dir.), Tourism Migration, Londres, Routledge, p.140.

8 Butler, R. (1980). « The Concept of a Tourist Area Cycle of Evolution », Canadian Geographer, vol. 24, n 1, p. 512. 


\section{Publicité}

4 programmes 